\title{
PARENTAL ORIGIN AND CELL STAGE ERRORS IN X-CHROMOSOME POLYSOMY 49,XXXXY
}

\author{
Guzel AI ${ }^{1, *}$, Demirhan $\mathrm{O}^{1}$, Pazarbasi $\mathrm{A}^{1}$, Yuksel B ${ }^{2}$
}

\begin{abstract}
*Corresponding Author: Ali Irfan Guzel, Department of Medical Biology and Genetics, Faculty of Medicine, Çukurova University, 01330 Adana, Turkey; Tel.: +90-322-338-70-68; Fax: +90322-338-70-65; E-mail: aliirfan@cu.edu.tr
\end{abstract}

\begin{abstract}
Polysomy $49, \mathrm{XXXXY}$ is a rare sex chromosome aneuploidy syndrome characterized by mental retardation, severe speech impairment, craniofacial abnormalities, multiple skeletal defects and genital abnormalities. We describe a patient with 49, XXXXY syndrome who had many characteristics of Fraccaro syndrome; language impairment, mongoloid slant, epicanthal folds, cryptorchidism, umbilical hernia and dysmyelinization in his brain. A GTG-banding technique was used for karyotype analysis of peripheral blood cell cultures. The parental origin of polysomy $\mathrm{X}$ was identified by using quantitative fluorescent polymerase chain reaction (QF-PCR) with seven short tandem repeat (STR) markers specific for the X/Y-chromosome which revealed that all the $\mathrm{X}$-chromosomes were of maternal origin. This report provides evidence for successive non disjunctions in maternal meiosis I and II.

Key words: Tetrasomy $\mathrm{X}$ syndrome; 49,XXXXY; Quantitative fluorescent polymerase chain reaction (QF-PCR); Short tandem repeat (STR) markers; Parental origin
\end{abstract}

1 Department of Medical Biology and Genetics, Faculty of Medicine, Çukurova University, Adana, Turkey

2 Department of Pediatrics, Faculty of Medicine, Çukurova University, Adana, Turkey

\section{INTRODUCTION}

The tetrasomy $\mathrm{X}(49, \mathrm{XXXXY})$ syndrome is a rare $\mathrm{X}$-chromosome polysomy with an estimated incidence of 1 in 85,000 male births [1]. The chromosomal constitution and clinical findings were described by Fraccaro et al. in 1960 [2]. Tetrasomy $\mathrm{X}$ has been considered as the most severe variant of Klinefelter's syndrome which is a common sex chromosomal abnormality caused by the presence of one additional $\mathrm{X}$ chromosome in males and has a prevalence of 1 in 500 [1-4]. The most common sex chromosome aberrations in live births are 47,XXY, 47,XXX, and 47,XYY [5]. However, rare syndrome variants with $\mathrm{X}$ and $\mathrm{Y}$ polysomy, mosaicisms and aberrant chromosomes have been reported, including 48,XXXY, 48,XXYY, 49XXXXY, 47,XXY/ 48,XXXY and 48,XXXY/49,XXXXY [6,7]. The clinical features of Klinefelter' syndrome are variable but often include infertility, gynecomastia, eunuchoidism, small testes and penis and hypergonadotropic hypogonadism. The extra $\mathrm{X}$ chromosome in these patients accounts for the clinical phenotype, but this ranges from mild (only infertility) to more severe with physical anomalies and mental retardation. The classical features of 49, XXXXY syndrome are growth and mental retardation, severe speech impairment, multiple skeletal defects, and dental, craniofacial and genital abnormalities. These patients have also phenotypic similarities such as strabismus, microcephaly, epicanthal folds, hypertelorism, cleft palate and heart disease 
$[2,8,9]$. We studied a patient with the $49, \mathrm{XXXXY}$ karyotype, verified and identified by quantitative fluorescent polymerase chain reaction (QF-PCR) amplification of seven short tandem repeat (STR) markers located on $\mathrm{X}$ and $\mathrm{Y}$ chromosomes, and two additional regions (AMXY for $\mathrm{X}$ and $\mathrm{Y}$, and SRY for Y chromosomes).

\section{PATIENT AND METHODS}

A 6-year-old boy, the first child of healthy and unrelated parents (mother 35 years old and father 40 years old), and had two healthy siblings. He was born after 40 weeks of normal gestation. Birth weight was $2,950 \mathrm{~g}$ and length $52 \mathrm{~cm}$. There was no family history of intellectual handicap or mental illness. He was referred to the Department of Pediatrics (Çukurova University, Adana, Turkey) because of development and speech delay concerns. He had patent ductus arteriosus in infancy, which had closed. No other sibling had similar features. There were no complications at birth but his psychomotor milestones were always behind those of his peers. He weighted $14 \mathrm{~kg}$ (45p for age, ' $p$ ' for percentile) and was $94 \mathrm{~cm}$ (45p for age) long. He was motor retarded, but showed no signs of aggressive behavior. He had mongoloid slant, epicanthal folds, cryptorchidism and umblical hernia, delayed tooth eruption and peg-shaped teeth. His hemogram, serum electrolytes and urine amino acids were normal. Magnetic resonance imaging of the brain showed no myelinization.

A GTG banding procedure was used for the analysis of metaphase chromosomes on peripheral blood cell cultures of the family. Twenty metaphases were analyzed microscopically.

Quantitative fluorescent PCR was used for identification of the parental origin of the extra $\mathrm{X}$ chromosomes. DNA was extracted from blood samples of the patient and his parents using InstaGene ${ }^{\mathrm{TM}}$ Matrix (Bio-Rad Laboratories, Hercules, CA, USA). The QF-PCR amplifications were performed using Aneufast ${ }^{\mathrm{TM}}$ (Molgentix SL, Barcelona, Spain) trisomy detection kit which includes fluorescently-labeled primers for 27 predefined STR marker sites on chromosomes 13, 18, 21, X (DXYS218, SBMA, DXS6803, DXS6809, HPRT, DXS8377 and X22) and Y (DXYS218 and X22), and primer pairs for AMXY (at Xp22.1-22.3 and
Yp11.2) and SRY (at Yp11.2) regions. The kit also contains dNTPs and HotStart Taq DNA polymerase in an optimized reaction buffer. Two $\mu$ of the DNA (5-10 ng) and $3 \mu \mathrm{L}$ of PCR-grade water were added to $10 \mu 1$ of each of the master mixes. After initial denaturation at $95^{\circ} \mathrm{C}$ for $15 \mathrm{~min}$., amplification was achieved by 28 cycles of $95^{\circ} \mathrm{C}$ for 40 seconds, $58^{\circ} \mathrm{C}$ for 80 seconds and $72^{\circ} \mathrm{C}$ for 40 seconds, and final extension was for $30 \mathrm{~min}$. at $60^{\circ} \mathrm{C}$. The QF-PCR products $(1.5 \mu \mathrm{L}$ from each mix) were added to $40 \mu \mathrm{L}$ Hi-Di' ${ }^{\mathrm{TM}}$ Formamide (Applied Biosystems, Foster City, CA, USA) containing $0.3 \mu \mathrm{L}$ of GeneScan ${ }^{\mathrm{TM}}-500$ LIZ $^{\mathrm{TM}}$ (Applied Biosystems) size standard. After denaturation at $95^{\circ} \mathrm{C}$ for $3 \mathrm{~min}$., the mixture was cooled to $4^{\circ} \mathrm{C}$ and then capillary electrophoresis was carried out on an ABI PRISM ${ }^{\mathrm{TM}} 310$ Genetic Analyzer (Applied Biosystems) using POP4 polymer. Analysis of the results was performed using GeneMapper 4.0 software (Applied Biosystems).

By means of the fluorescent primers, the amplified segments could be visualized and quantified as peak areas on automated DNA scanners. As the amount of PCR product was proportional to the initial amount of target DNA, normal heterozygous subjects were expected to show two equal peak areas (ratio 1:1) for each chromosome analyzed. In the case of aneuploidy of a chromosome, the ratio would change according to the change in number of a chromosome $[10,11]$.

The parental origin of the aneuploidy was determined by comparision of the STR alleles of the patient and his parents. Meiotic division errors in meiosis I or meiosis II were inferred on the basis of non reduction/reduction stage of the chromosome by comparision of the proximal (pericentromeric) markers. If parental heterozygosity was retained in the aneuploidic child, we concluded that the error occurred during meiosis I, and if parental heterozygosity was reduced to homozygosity, we concluded that the error occurred during meiosis II or in post-zygotic mitosis. Mitotic errors were distinguished from meiosis II by evaluation of medial and distal markers. If homozygosity was not observed at all informative loci, including at least one each in proximal, medial, and distal portions of the chromosome, a post-zygotic origin was inferred. If homozygosity was observed at one or more loci, the error was assigned to meiosis II [11-15]. 


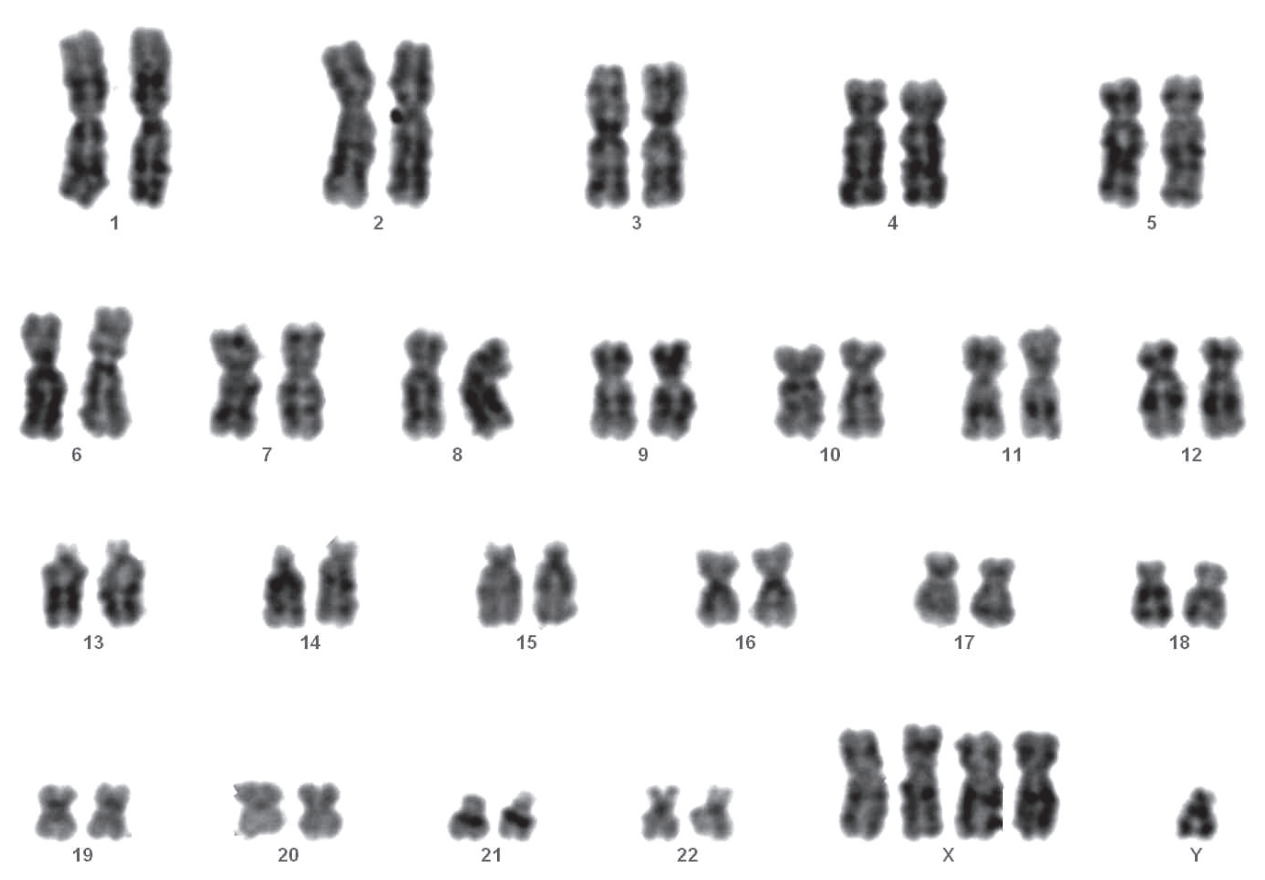

Figure 1. G-banded metaphase image showing chromosome X tetrasomy in the patient.

\section{RESULTS}

While mother, father and brothers had normal karyotypes, the patient had a 49,XXXXY chromosome constitution (Figure 1). We inferred that the polysomy appeared to be due to de novo non disjunction of the X-chromosomes. After evaluation of nine regions specific to $\mathrm{X}$ and $\mathrm{Y}$ chromosomes, the origin of the extra chromosomes was revealed. Comparison of the area of the peaks (Figure 2) for the patient and his parents showed an imbalance between his $\mathrm{X}$ and $\mathrm{Y}$ chromosome in the AMXY and DXYS218 regions (ratios 4:1), indicating his tetra $X$ constitution. All X chromosome-specific STR markers of the patient were inherited from his mother and heterozygosity of these markers was retained at proximal, medial and distal regions of the extra chromosomes. These are the expected outcomes for non disjunctions in both M I and M II.

\section{DISCUSSION}

The 49,XXXXY syndrome has not been reported to be associated with maternal age or a hereditary disease [4]. The extra X chromosomes result sporadically from either meiotic non disjunction where a chromosome fails to separate during the first or second division of gametogenesis or from mitotic non disjunction in the developing zygote. The likelihood of $\mathrm{X}$ chromosome non disjunction increases with advancing maternal age. It is believed the that $49, \mathrm{XXXXY}$ syndrome occurs during maternal non disjunction both in meiosis I and II $[15,16]$. In our patient, all the $\mathrm{X}$ chromosome-specific STR markers we tested for were inherited from his mother and heterozygosity was retained in all regions (proximal, medial and distal regions) of all. These are the expected conditions of non disjunction in both meiosis I and meiosis II. Molecular genetic analyses using polymorphic DNA markers have enabled the study of non disjunction of fetal aneuploidy and the determination of parental origin and cell stage errors at meiosis I or II [11,13-15].

The common presentations of the 49, XXXXY syndrome during early infancy have included variable combinations of craniofacial dysmorphism, abnormal genitals and delayed developmental milestones that lead to chromosome karyotyping $[4,9,18$ 20]. Our patient had classical facial features of the 49 ,XXXXY syndrome. Whereas coarsening of facial features has been reported to occur later in life [19], these were evident very early in our patient. Also newborns with the 49, XXXXY syndrome were reported to have below average lengths and weights at 


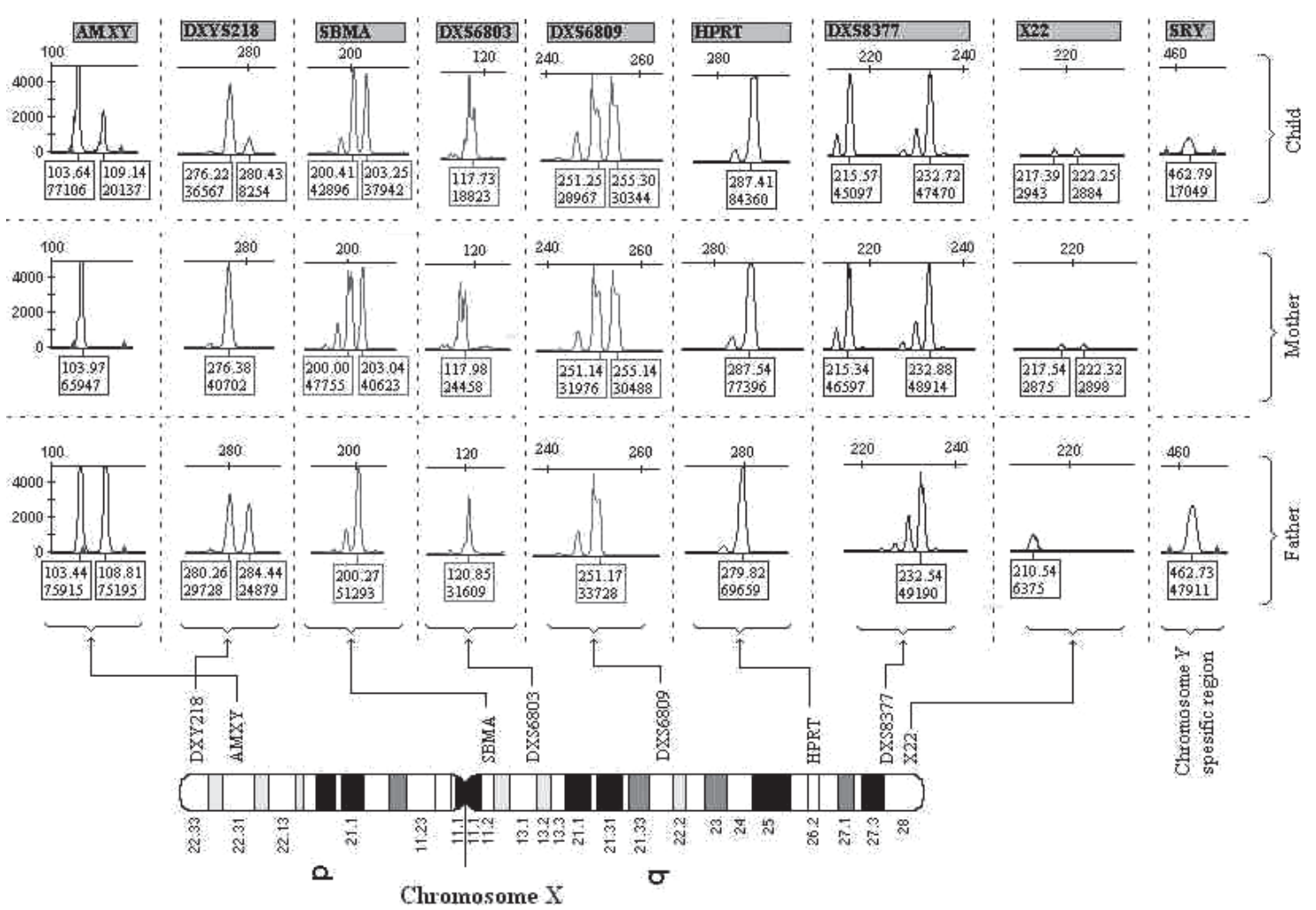

Figure 2. Electrophoretograms of the QF-PCR products of six microsatellite markers on chromosomes X (SBMA, DXS6803, DXS6809 and HPRT) and Y (DXYS218 and X22) of the patient and his parents. AMXY (on chromosome $\mathrm{X}$ and $\mathrm{Y}$ ) and SRY (only on chromosome $\mathrm{Y}$ ) region were used for the identification and comparison of the number of $\mathrm{X}$ and $\mathrm{Y}$ chromosomes. The box under each peak includes molecular size (bp) and area of the peak. Smaller peaks in front (from left to right) of some peaks are stutter peaks and should be discounted. Ratios of the peak areas of AMXY and DXYS218 are 3.8:1 and 4.4:1 respectively (approximately 4:1) in the patient and indicate the presence of four X and one Y chromosomes. All chromosome X markers of the patient were inherited from the X chromosomes of the mother.

birth, and demonstrated significant catch-up growth in later gestation $[4,20]$. In our patient, the growth parameters were appropriate for his gestational age, but he failed to thrive thereafter. Multiple skeletal anomalies, with radioulnar synostosis in particular, present in our patient, are characteristic of the 49, XXXXY syndrome $[4,18]$. The addition of more $\mathrm{X}$ chromosomes in polysomy $\mathrm{X}$ males results not only in infertility, but also in hypoplastic and undervirilized genitalia [18]. The majority of patients with 48, XXXY and 49, XXXXY syndromes have been reported to have small testes (94\%), small penis and hypoplastic scrotum (80\%) cryptochidism (30\%) and ambiguous genitalia [21]. Mental retardation is anticipated to be a major problem in this patient. A direct relationship between the number of supernumerary X chromosomes with phenotypic abnormali- ties and mental retardation has been reported, the severity of mental retardation increasing with each additional $\mathrm{X}$ chromosome [18,21]. Patients with 49, XXXXY syndrome have varying degrees of mental retardation [22]. Our patient has developmental delay and showed speech and articulation problems. These findings are in agreement with the important delay in language development and reading difficulties before adolescence that has been seen in patients with sex-chromosomal abnormalities [23,24].

We conclude that the 49,XXXXY syndrome is different from the Klinefelter's syndrome in many ways. Chromosome karyotyping is warranted in the presence of facial dysmorphism or other somatic abnormalities, to exclude underlying sex chromosomal aneuploidy disorders such as the 49, XXXXY syndrome. Evaluation of exceptional instances of segre- 
gation failure may be useful in improving our understanding of the general mechanisms of non disjunction. Molecular techniques could be useful for the pre- and post-natal diagnosis of the common aneuploidies and for determining their parental origin. This kind of study will improve knowledge about the mechanisms of the development of aneuploidies, and provide an opportunity to study the effect of the 49 , XXXXY syndrome on the phenotype, and enable appropriate and rapid genetic counseling.

\section{REFERENCES}

1. Visootsak J, Graham JM Jr. Klinefelter syndrome and other sex chromosomal aneuploidies. Orphanet J Rare Dis 2006; 1: 42.

2. Fraccaro M, Kaijser K, Lindsten J. A child with 49 chromosomes. Lancet 1960; 2(7156): 899902.

3. Jacobs PA, Strong JA. A case of human intersexuality having possible XXY sex-determining mechanism. Nature 1959; 183(4657): 164-167.

4. Peet J, Weaver DD, Vance GH. 49,XXXXY: a distinct phenotype. Three new cases and review. J Med Genet 1988; 35(5): 420-424.

5. Thompson MW, McInnes RR, Huntington WF, Eds. Thompson \& Thompson Genetics in Medicine, 5 ed. Philadelphia: W.B. Saunders Company. 1991; 172-175.

6. Hirschhorn K, Hirschhorn R, Fraccaro M, Book JA. Incidence of familial hyperlipemia. Science $1959 ;$ 129(3350): 716-717.

7. Demirhan O. Clinical findings and phenotype in a toddler with 48,XXYY syndrome. Am J of Med Genet 2003; 119A(3): 393-394.

8. Griffin JE, Wilson JD. Disorders of the testes and male reproductive tract. In: Wilson JD, Foster DW, Eds. Williams Text Book of Endocrinology. Philadelphia: W.B. Saunders Company. 1992; 884884.

9. Lia EN, Otero SAM, Ferraz M, Gonçalves LPV. Oral aspects of 49, XXXXY syndrome: a case report. J Dentist Childr 2007; 74(2): 136-139.

10. Adinolfi M, Pertl B, Sherlock J. Rapid detection of aneuploidies by microsatellite and the quantitative fluorescent polymerase chain reaction. Prenat Diagn 1997; 17(13): 1299-1311.

11. Diego-Alvarez D, Ramos-Corrales C, Garcia-Hoyos M, Bustamante-Aragones A, Cantalapie- dra D, Diaz-Recasens J, Vallespin-Garcia E, Ayuso C, Lorda-Sanchez I. Double trisomy in spontaneous miscarriages: cytogenetic and molecular approach. Hum Reprod 2006; 21(4): 958-966.

12. Lamb NE, Freeman SB, Savage-Austin A, Pettay D, Taft L, Hersey J, Gu Y, Shen J, Saker D, May KM, Avramopoulos D, Petersen MB, Hallberg A, ikkelsen M, Hassold TJ, Sherman SL. Susceptible chiasmate configurations on chromosome 21 predispose to non-disjunction in both maternal meiosis I and meiosis II. Nat Genet 1996; 14(4): 400405 .

13. Nicolaidis P, Petersen MB. Origin and mechanisms of nondisjunction in human autosomal trisomies. Hum Reprod 1998; 13(2): 313-319.

14. Robinson WP, Bernasconi-Quadroni F, Lau A, McFadden DE. Frequency of meiotic trisomy depends on involved chromosome and mode of ascertainment. Am J Med Genet 1999; 84(1): 34-42.

15. Guzel AI, Demirhan O, Pazarbasi O, Ozgunen FT, Kocaturk-Sel S, Tastemir D. Detection of parental origin and cell stage errors of a double nondisjunction in a fetus by QF-PCR. Genet Test Molec Biomark 2009; 13(1): 73-77.

16. Celik A, Eraslan S, Gokgoz N, Ilgin H, Basaran S, Bokesoy I, Kayserili H, Yuksel-Apak M, Kirdar Betul. Identification of the parental origin of polysomy in two $49, \mathrm{XXXXY}$ cases. Clinic Genet 1997; 51(6): 426-429.

17. Deng H-X, Abe K, Kondo I, Tsukahara M, Inagaki H, Hamada Isamu, Fukushima Yoshimitsu and Niikawa N. Parental origin and mechanism of formation of polysomy $\mathrm{X}$ : an XXXXX case and four XXXXY cases determined with RFLPs. Hum Genet 1986; 86(6): 541-544.

18. Linden MG, Bender BG, Robinson A. Sex chromosome tetrasomy and pentasomy. Pediatrics 1995; 96(4, Pt. 1):672-682.

19. Sijmons RH, van Essen AJ, Visser JD, Iprenburg M, Nelck GF, Vos-Bender ML, de Jong B. Congenital knee dislocation in a 49, XXXXY boy. J Med Genet 1995; 32(4): 309-311.

20. Hayek A, Riccardi V, Atkins L, Hendren H. $49, X X X X Y$ chromosomal anomaly in a neonate. $\mathrm{J}$ Med Genet 1971; 8(2): 220-221.

21. Jones KL. XXY syndrome, Klinefelter syndrome. In: Jones KL, Ed. Smith's Recognizable Patterns of Human Malformation, 5th ed. Philadelphia: W.B. Saunders. 1997; 72-77. 
22. Borghgraef M, Fryns JP, Van Den Berghe H. The 48,XXYY syndrome: follow-up data on clinical characteristics and psychological finding in 4 patients. Genet Counsel 1991; 2(2): 103-108.

23. Garvey M, Mutton DE. Sex chromosome observations and speech development. Arch Dis

\section{Child 1973; 48(12): 937-941.}

24. Steward DA, Bailey JD, Netley CT, Rovet J, Park E. Growth and development of children with $\mathrm{X}$ and $\mathrm{Y}$ chromosome aneuploidy from infancy to pubertal age; the Toronto study. Birth Defects Orig Artic Ser 1982; 18(4): 99-154. 